Tropical Journal of Pharmaceutical Research October 2019; 18 (10): 2067-2074

ISSN: $1596-5996$ (print); 1596-9827 (electronic)

(C) Pharmacotherapy Group, Faculty of Pharmacy, University of Benin, Benin City, 300001 Nigeria.

\title{
Network pharmacology-based elucidation of the molecular mechanism underlying the anti-migraine effect of Asari Radix et Rhizoma
}

\author{
Yun-Bin Jiang ${ }^{1 *}$, Mei Zhong ${ }^{1}$, Ting Huang ${ }^{2}$, Zhong-Hua Dai ${ }^{3}$, Xing-Bao Tao ${ }^{4}$, \\ Rong-Ping Yang ${ }^{1}$ \\ ${ }^{1}$ College of Pharmaceutical Sciences and Chinese Medicine, Southwest University, Chongqing $400715,{ }^{2}$ College of Pharmacy, \\ Chengdu University of Traditional Chinese Medicine, Chengdu 611137, ${ }^{3}$ College of Pharmacy, Guangxi University of Chinese \\ Medicine, Nanning 530001, ${ }^{4}$ College of Pharmacy, Nanjing University of Chinese Medicine, Nanjing 210023, PR China
}

${ }^{*}$ For correspondence: Email: yunbinjiang@swu.edu.cn

Sent for review: 11 May 2019

Revised accepted: 27 September 2019

\begin{abstract}
Purpose: To determine the molecular mechanism involved in the anti-migraine effect of Asari Radix et Rhizoma (ARR) using network pharmacology.

Methods: The compounds present in ARR were identified through information retrieval from literature and public databases, and were screened based on absorption, distribution, metabolism, excretion and toxicity. Target genes related to the selected compounds and migraine were identified or predicted from public databases. Hub genes in ARR against migraine were identified through analysis of interactions in overlapping genes between compounds and migraine target genes, based on STRING database. Gene enrichment analysis of overlapping genes was performed using Database for Annotation, Visualization and Integrated Discovery.

Results: A total of 138 compounds were selected as potential bioactive compounds in ARR. Target genes related to the selected compounds (611 genes) and migraine (278 genes) were obtained, including 71 overlapping genes. The hub genes in the anti-migraine effect of ARR were BDNF, IL6, COMT, APP and TNF. Gene enrichment analysis showed the top 10 biological processes or pathways involved in the mechanism of anti-migraine action of ARR. The tissue source of the overlapping genes was not limited to the brain. The results from gene enrichment analysis revealed that the effect of $A R R$ on migraine was holistic, which is characteristic of traditional Chinese medicines.

Conclusion: Network pharmacology has been used to decipher the molecular mechanism involved in the action of ARR against migraine. The results provide a scientific basis for the clinical effect of ARR on migraine.
\end{abstract}

Keywords: Asari Radix et Rhizoma, Migraine, Network pharmacology, Molecular mechanism, Hub genes

This is an Open Access article that uses a fund-ing model which does not charge readers or their institutions for access and distributed under the terms of the Creative Commons Attribution License (http://creativecommons.org/licenses/by/4.0) and the Budapest Open Access Initiative (http://www.budapestopenaccessinitiative.org/read), which permit unrestricted use, distribution, and reproduction in any medium, provided the original work is properly credited.

Tropical Journal of Pharmaceutical Research is indexed by Science Citation Index (SciSearch), Scopus, International Pharmaceutical Abstract, Chemical Abstracts, Embase, Index Copernicus, EBSCO, African Index Medicus, JournalSeek, Journal Citation Reports/Science Edition, Directory of Open Access Journals (DOAJ), African Journal Online, Bioline International, Open-J-Gate and Pharmacy Abstracts

\section{INTRODUCTION}

Migraine is a chronic and sometimes progressive disease characterized by neurological disorders resulting in recurrent episodes of moderate or severe head pain and a myriad of neurological symptoms. Patients with migraine often have some characteristic symptoms such as 
gastrointestinal symptoms e.g. emesis and nausea, photophobia, cutaneous allodynia, and phonophobia [1]. Moreover, migraine patients bear other neurological symptoms such as tinnitus, vertigo, and cognitive impairment [2]. Migraine exerts enormous economic burden and mental stress on the patient and the society at large [3]. Thus, there is need to develop safe and effective anti-migraine drugs.

Although anti-migraine western drugs have been developed all over the world [4], traditional Chinese medicines (TCMs) have also been widely used to treat migraine in China [5]. Asari Radix et Rhizoma (ARR), composed from the roots of Asarum heterotropoides Fr. Schmidt var. mandshuricum (Maxim.) Kitagawa, A. sieboldii Miq. var. seoulense, and $A$. sieboldii Miq., exhibits a wide range of pharmacological effects such as analgesic, sedative, anticonvulsant, hypotensive, anti-aging, and anti-inflammatory properties [6]. It is usually used as an herbal medicine to treat migraine in China $[7,8]$. However, not much is known about the molecular mechanisms underlying the anti-migraine effect of ARR. It is necessary to elucidate the mechanisms of anti-migraine action of ARR so as to rationally guide its clinical use.

With the rapid development of systems, network, and chemical biology, network pharmacology has emerged as a promising strategy in current drug discovery and development. It is used to study diseases from "network targets, multicomponent therapeutics" instead of "one target, one drug" [9]. This characteristic is consistent with the concept of holism. Coincidentally, the perspective of holism has long been central to TCMs treatments of various diseases [10]. Therefore, network pharmacology is a valuable tool for achieving holistic view of TCMs. The current TCMs issues based on how to study TCMs from point to face could be tackled with network pharmacology. Nowadays, network pharmacology is used widely to achieve comprehensive and systematic insight into the bioactive compounds and mechanisms of action of TCMs [11]. The aim of this study was to use network pharmacology to determine the molecular mechanism involved in the antimigraine effect of ARR.

\section{EXPERIMENTAL}

\section{Compounds collection and ADMET evaluation}

The compounds present in ARR were identified from existing literature and three public databases: Symptom Mapping (SymMap, http://www.symmap.org/), Traditional Chinese Medicine Systems Pharmacology Database and Analysis Platform (TCMSP, http://lsp.nwu.edu.cn/ tcmsp.php), Encyclopedia of Traditional Chinese Medicine (ETCM, http://www.nrc.ac.cn:9090/ ETCM/). PubChem CID, chemical structures, and SMILES of each compound were identified with PubChem (https://pubchem.ncbi.nlm.nih.gov/). All compounds were screened using ADMET evaluation with the aid of FAFDrugs4 (http://fafdrugs4.mti.univ-paris-diderot.fr/). The "PhysChem Filters" parameter of FAFDrugs 4 was set as "Drug-Like Soff". Compounds were retained only if the result of ADMET evaluation was "Accepted".

\section{Target genes related to the selected compounds}

Target genes linked to the identified compounds were collected from the databases SymMap, TCMSP, ETCM and Therapeutic Target Database (TTD, http://bidd.nus.edu.sg/group/cjttd/), and were predicted by STITCH (http://stitch.embl.de/) and SwissTargetPrediction (http://swisstargetpredic tion.ch/) with "Homo sapiens" setting. When the information obtained was a protein name, UniProt (https://www.uniprot.org/) was used to transfer the protein name to gene symbol with "Homo sapiens" setting.

\section{Target genes related to migraine}

Migraine-related target genes were identified by retrieving Online Mendelian Inheritance in Man (OMIM, https://omim.org/), DisGeNET (www. disgenet.org/), TTD and TCMSP databases. The overlapping genes were identified by matching compounds-related target genes with migrainerelated target genes with the aid of a Venn diagram plotted using the OmicShare tools, a free online platform for data analysis (www.omicshare.com/tools).

\section{Protein-protein interaction (PPI) network analysis}

The PPI network analysis of the overlapping genes was carried out on STRING (https://string$\mathrm{db}$. org/) with "Homo sapiens" setting. The constructed PPI network of overlapping genes was visualized using Cytoscape ver. 3.7.1 (https://cytoscape.org/). Nodes in networks represent genes, while edges suggest interactions among genes. The hub genes involved in the action of ARR against migraine were selected by setting threshold value of Degree in network. In this analysis, the bigger 
the Degree values of genes, the more important the genes.

\section{Gene enrichment analysis}

Gene enrichment analysis of the overlapping genes was performed using Database for Annotation, Visualization and Integrated Discovery ver. 6.8 (https://david.ncifcrf.gov/) with "Homo sapiens" setting. The results of gene ontology (GO)-biological process, Kyoto Encyclopedia of Genes and Genomes (KEGG) pathway, and tissue expression enrichment analyses were used to unravel the molecular mechanism involved in the anti-migraine effect of ARR. Bubble charts of GO-biological process and KEGG pathways of the overlapping genes were plotted using the OmicShare tools.

\section{RESULTS \\ Compounds present in ARR and ADMET evaluation}

A total of 259 compounds from ARR were identified through retrieval from extant literature, SymMap, TCMSP, and ETCM. Moreover, PubChem CID, chemical structures, and SMILES of each compound were confirmed through PubChem. Then, the SMILES of all compounds were imported into FAFDrugs4 for ADMET evaluation. The results suggested that 138 compounds exhibited good potential as active ingredients.

Target genes related to the 138 compounds and migraine

A total of 611 genes linked to the 138 compounds were identified through information retrieval from SymMap, TCMSP, ETCM, TTD, STITCH and SwissTargetPrediction databases. Genes related to migraine (278 genes) were collected from OMIM, DisGeNET, TTD and TCMSP databases. The Venn diagram shown in Figure 1 indicates that 71 overlapping genes were identified by matching the 611 genes related to the 138 compounds with the 278 genes related to migraine. The gene symbol and UniProt ID of 71 overlapping genes are listed in Table 1.

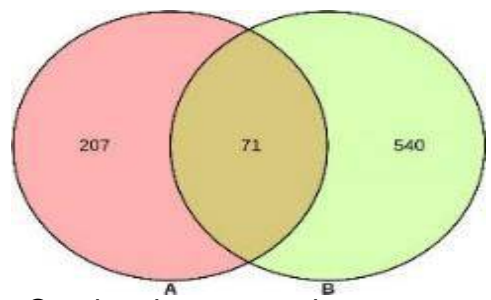

Figure 1: Overlapping genes between target genes related to migraine $(A)$ and compounds in ARR $(B)$
Table 1: Gene symbol and UniProt ID of 71 overlapping genes

\begin{tabular}{|c|c|c|}
\hline No. & Gene symbol & UniProt ID \\
\hline 1 & SLC6A2 & P23975 \\
\hline 2 & NOS2 & P35228 \\
\hline 3 & SLC6A4 & P31645 \\
\hline 4 & MMP3 & P08254 \\
\hline 5 & HTR4 & Q13639 \\
\hline 6 & GRM2 & Q14416 \\
\hline 7 & HTR2B & P41595 \\
\hline 8 & CCR1 & P32246 \\
\hline 9 & MAOB & P27338 \\
\hline 10 & CYP1A2 & P05177 \\
\hline 11 & TRPM8 & Q7Z2W7 \\
\hline 12 & BACE1 & P56817 \\
\hline 13 & CYP2D6 & P10635 \\
\hline 14 & GSTM1 & P09488 \\
\hline 15 & NOS1 & P29475 \\
\hline 16 & IL10 & P22301 \\
\hline 17 & CA14 & Q9ULX7 \\
\hline 18 & GNAS & O95467 \\
\hline 19 & HTR2C & P28335 \\
\hline 20 & MTRR & Q9UBK8 \\
\hline 21 & DRD5 & P21918 \\
\hline 22 & MAOA & P21397 \\
\hline 23 & BDNF & P23560 \\
\hline 24 & KCNA3 & P22001 \\
\hline 25 & DRD3 & P35462 \\
\hline 26 & DAO & P14920 \\
\hline 27 & F7 & P08709 \\
\hline 28 & EDNRA & P25101 \\
\hline 29 & DRD4 & P21917 \\
\hline 30 & NOS3 & P29474 \\
\hline 31 & $\mathrm{DDC}$ & P20711 \\
\hline 32 & GSTP1 & P09211 \\
\hline 33 & GABRQ & Q9UN88 \\
\hline 34 & CNR1 & P21554 \\
\hline 35 & PGR & P06401 \\
\hline 36 & SCN1A & P35498 \\
\hline 37 & TLR4 & 000206 \\
\hline 38 & CYP19A1 & P11511 \\
\hline 39 & GC & P02774 \\
\hline 40 & ADRB1 & P08588 \\
\hline 41 & TNF & P01375 \\
\hline 42 & CCR2 & P41597 \\
\hline 43 & CACNA1H & 095180 \\
\hline 44 & $\mathrm{ADH} 1 \mathrm{~B}$ & P00325 \\
\hline 45 & LDLR & P01130 \\
\hline 46 & DRD2 & P14416 \\
\hline 47 & TYRP1 & P17643 \\
\hline 48 & $\mathrm{~F} 2$ & P00734 \\
\hline 49 & TRPV3 & Q8NET8 \\
\hline 50 & TRPA1 & 075762 \\
\hline 51 & PTGS2 & P35354 \\
\hline 52 & IL1B & P01584 \\
\hline 53 & REN & P00797 \\
\hline 54 & ESR1 & P03372 \\
\hline 55 & HTR2A & P28223 \\
\hline 56 & CSNK1D & P48730 \\
\hline 57 & ADRB2 & P07550 \\
\hline 58 & AR & P10275 \\
\hline 59 & ESR2 & Q92731 \\
\hline 60 & GABRA3 & P34903 \\
\hline 61 & CCL2 & P13500 \\
\hline 62 & CYP3A4 & P08684 \\
\hline 63 & IL6 & P05231 \\
\hline
\end{tabular}

Trop J Pharm Res, October 2019; 18(10):2069 
Table 1: Gene symbol and UniProt ID of 71 overlapping genes (continued)

\begin{tabular}{lll}
\hline No. & Gene symbol & UniProt ID \\
\hline & & \\
\hline 64 & SCN5A & Q14524 \\
65 & GRIA2 & P42262 \\
66 & APP & P05067 \\
67 & COMT & P21964 \\
68 & SLC6A3 & Q01959 \\
69 & TRPV1 & Q8NER1 \\
70 & MMP9 & P14780 \\
71 & DRD1 & P21728 \\
\hline
\end{tabular}

Hub genes involved in the action of ARR against migraine

As shown in Figure 2, the PPI network of overlapping genes consisted of 69 nodes and 487 edges. Based on Degrees of genes, the top 5 genes (BDNF, IL6, COMT, APP and TNF) were considered as the hub genes involved in the action of ARR against migraine. This indicated that these genes play important roles in the molecular mechanisms involved in the antimigraine effect of ARR.

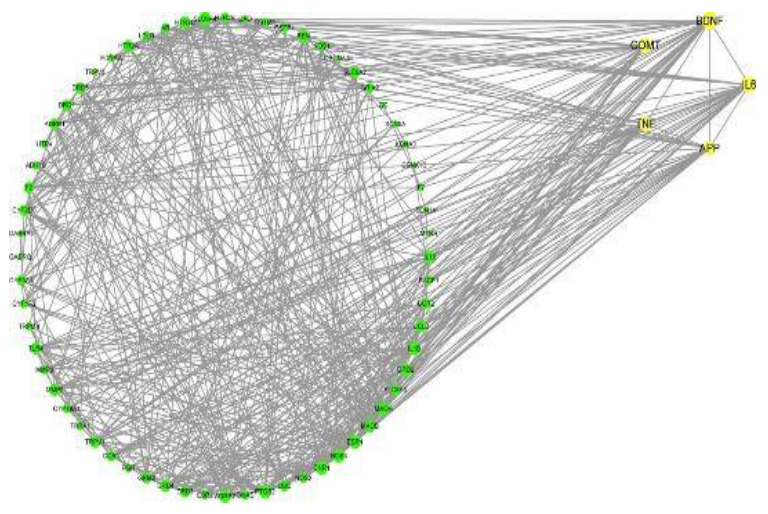

Figure 2: PPI network of overlapping genes including 69 nodes and 487 edges. Yellow nodes: hub genes involved in the anti-migraine effect of ARR

\section{Gene enrichment analysis of 71 overlapping genes}

The results of biological process enrichment analysis indicated that 265 biological processes were related to the anti-migraine effect of ARR, and the top 10 biological processes were response to drug, cellular calcium ion homeostasis, negative regulation of blood pressure, positive regulation of nitric oxide biosynthetic process, synaptic transmission, dopaminergic, behavioral response to cocaine, activation of adenylate cyclase activity, positive regulation of ERK1 and ERK2 cascade, dopamine catabolic process, and positive regulation of fever generation. The results are shown in Figure 3 and listed in Table 2.
The results of KEGG pathway enrichment analysis suggested that 52 pathways were related to the anti-migraine effect of ARR, and the top 10 pathways were neuroactive ligandreceptor interaction, cocaine addiction, dopaminergic synapse, serotonergic synapse, calcium signaling pathway, drug metabolismcytochrome P450, inflammatory mediator regulation of TRP channels, tyrosine metabolism, gap junction, and amphetamine addiction. The results are shown in Figure 4 and listed in Table 3.

The results of tissue expression analysis suggested that the main tissue source of 71 overlapping genes was the brain (38 genes), and some other tissues were involved, including liver, blood, heart, breast, leukocyte, fibroblast, brain stem, G-protein coupled receptors, and breast cancer. The results are presented in Table 4.

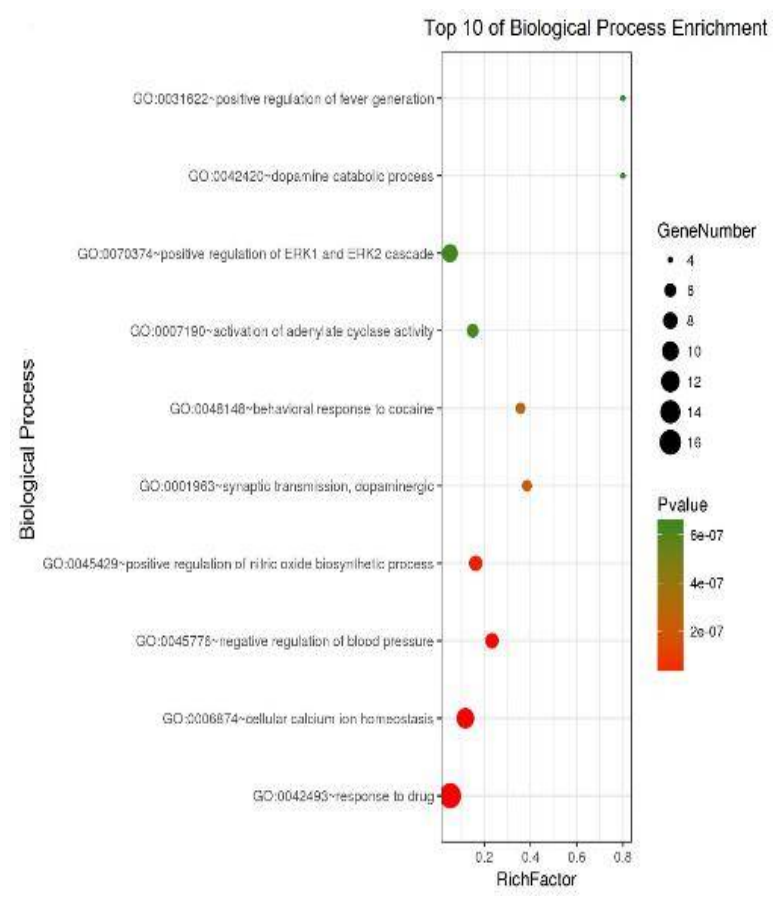

Figure 3: Bubble chart of the top 10 of GO-biological processes of 71 overlapping genes

\section{DISCUSSION}

In the present study, the molecular mechanism involved in the anti-migraine effect of ARR was systematically unraveled using network pharmacology. The results showed the hub genes, biological processes, and molecular pathways through which ARR acts against migraine. The tissue expressions of genes related to ARR action were also analyzed. 
Table 2: Target genes in the top 10 of GO-biological processes

\begin{tabular}{ll}
\hline GO ID & Target genes \\
\hline GO:0042493 & IL6, DRD1, DRD3, PTGS2, DRD2, SLC6A2, SLC6A3, SLC6A4, TRPA1, MAOB, GNAS, COMT, \\
GO:0006874 & HTR2B, HTR2C, IL10, HTR2A \\
GO:0045776 & NOS1, DRD3, DRD2, DRD5, CNR1, NOS3, NOS2 \\
GO:0045429 & IL6, TNF, PTGS2, TRPV1, ESR1, IL1B, TLR4 \\
GO:0001963 & DRD1, DRD3, DRD2, DRD5, DRD4 \\
GO:0048148 & DRD1, DRD3, DRD2, DRD4, HTR2A \\
GO:0007190 & EDNRA, DRD1, ADRB2, ADRB1, DRD5, GNAS \\
GO:0070374 & IL6, TNF, CCL2, DRD2, CCR1, TLR4, HTR2B, HTR2C, HTR2A \\
GO:0042420 & SLC6A3, MAOA, MAOB, COMT \\
GO:0031622 & TNF, PTGS2, CNR1, IL1B \\
\hline
\end{tabular}

Table 3: Target genes in the top 10 of KEGG pathways

\begin{tabular}{ll}
\hline Pathway ID & Target genes \\
\hline hsa04080 & DRD1, DRD3, TRPV1, DRD2, GABRA3, DRD5, DRD4, HTR4, EDNRA, ADRB2, \\
hsa05030 & GRM2, ADRB1, GRIA2, CNR1, F2, HTR2B, HTR2C, GABRQ, HTR2A \\
hsa04728 & DDC, DRD1, BDNF, GRM2, GRIA2, DRD2, SLC6A3, MAOA, MAOB, GNAS \\
& DDC, DRD1, SCN1A, DRD3, GRIA2, DRD2, SLC6A3, DRD5, MAOA, DRD4, MAOB, \\
hsa04726 & GNAS, COMT \\
& DDC, APP, PTGS2, MAOA, SLC6A4, MAOB, CYP2D6, HTR4, GNAS, HTR2B, \\
hsa04020 & DRR2C, HTR2A \\
hsa00982 & NOS2, HTR2B, HTR2C, HTR2A \\
hsa04750 & GSTM1, CYP3A4, MAOA, MAOB, CYP2D6, ADH1B, CYP1A2, GSTP1 \\
hsa00350 & TRPM8, TRPV1, TRPV3, TRPA1, IL1B, GNAS, HTR2B, HTR2C, HTR2A \\
hsa04540 & DDC, TYRP1, MAOA, MAOB, ADH1B, COMT \\
hsa05031 & DRD1, ADRB1, CSNK1D, DRD2, GNAS, HTR2B, HTR2C, HTR2A \\
\hline
\end{tabular}

Table 4: Tissue expression analysis of 71 overlapping genes

\begin{tabular}{ll}
\hline Tissues & Target genes \\
\hline Breast & CYP3A4, ESR1, GNAS, SCN5A \\
Leukocyte & APP, ADRB2, BDNF, IL1B \\
Blood & AR, TYRP1, ADRB2, TNF, CCR1, MMP9, F2, KCNA3 \\
& DRD1, SCN1A, LDLR, CA14, DRD3, TRPV1, DRD2, SLC6A2, DRD5, \\
Brain & SLC6A3, CYP2D6, SLC6A4, DRD4, ADH1B, KCNA3, COMT, EDNRA, \\
& BDNF, APP, CNR1, DAO, SCN5A, AR, NOS1, GABRA3, MAOB, ESR1, \\
& HTR4, ADRB2, GRM2, CSNK1D, GRIA2, BACE1, GNAS, HTR2B, \\
Brain stem & HTR2C, GSTP1, HTR2A \\
G-protein coupled receptors & CNR1, HTR2A \\
Heart & CCR2, HTR4 \\
Breast cancer & GSTM1, ADRB2, CA14, HTR4, CACNA1H, SCN5A \\
Liver & PGR, ESR1 \\
Fibroblast & CYP3A4, TYRP1, LDLR, MAOA, CYP2D6, ADH1B, CYP1A2, F7, \\
\hline
\end{tabular}

The ADMET evaluation serves to predict the bioactive potential of target compounds [12]. In this study, ADMET evaluation of 259 compounds present in ARR was carried out on FAFDrugs4 platform prior to searching target genes of the compounds. The results revealed that 138 compounds exhibited good potential as active ingredients.

STRING is an accepted database used to construct PPI networks of multiple genes [13]. In this study, PPI network of the 71 overlapping genes showed the degree of contribution of each gene to the mechanism involved in the antimigraine action of ARR, while BDNF, IL6, COMT, APP and TNF were identified as the hub genes. The BDNF is an important regulatory factor of pain, and many investigations have indicated that it exerts an important influence on migraine pathophysiology [14]. It has been reported that migraine is positively associated with TNF- $\alpha$, and negatively associated with IL-6 [15]. Some studies investigated the relationship between genetic polymorphisms of COMT and susceptibility to migraine, but the results were 


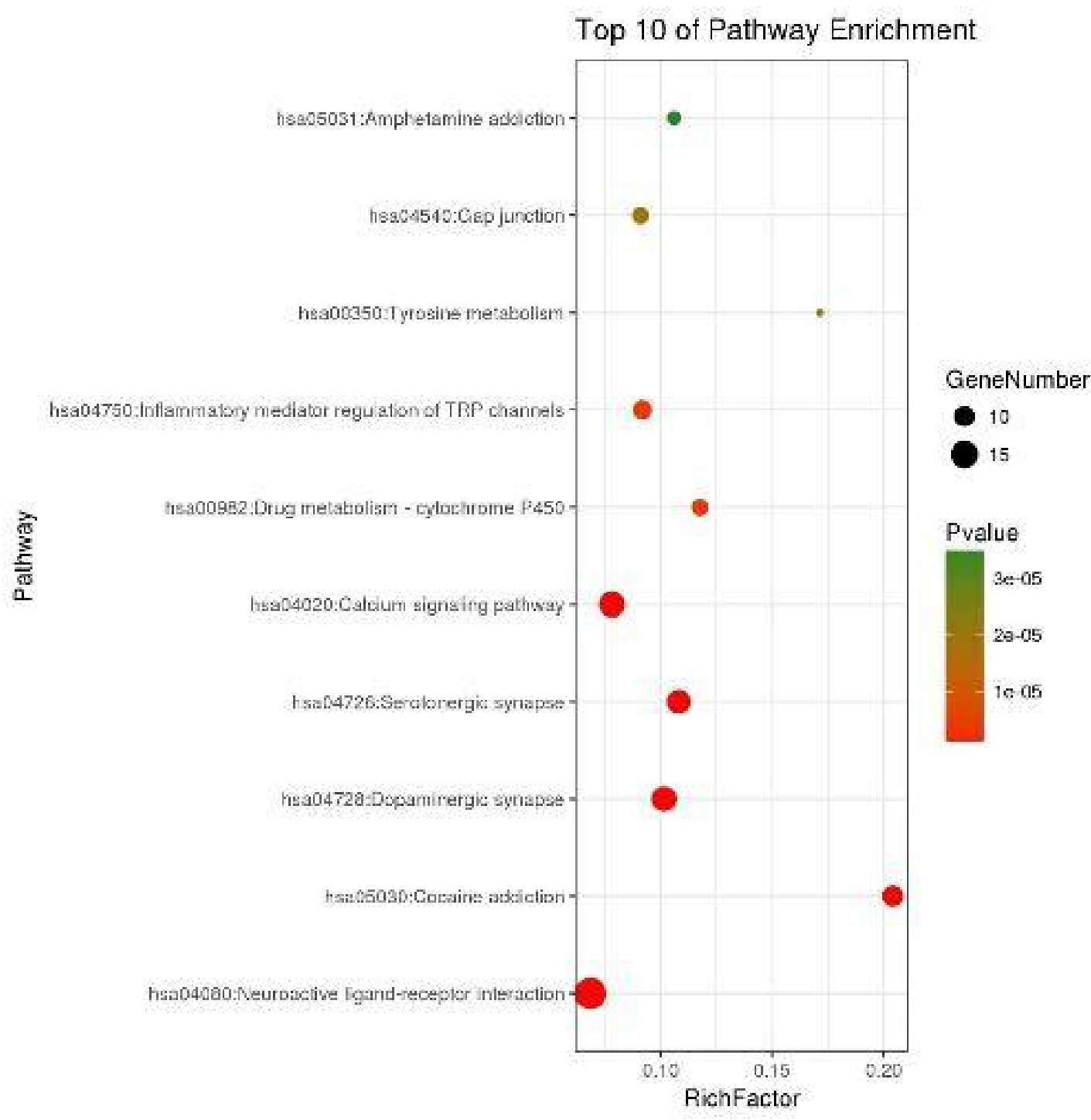

Figure 4: Bubble chart of the top 10 of KEGG pathways of 71 overlapping genes

inconsistent [16,17]. However, meta-analysis has indicated that COMT polymorphisms might decrease the risk of migraine [18]. Increases in levels of amyloid $\beta$-protein (the hydrolysate of amyloid- $\beta$ precursor protein) suppresses the occurrence of migraine by inhibiting the activity of $\mathrm{Na}+-\mathrm{K}+-\mathrm{ATPase}$ in capillary endothelial cells, resulting in suppression of cortical spreading depression [19]. These reports indicate that the 5 hub genes (BDNF, IL6, COMT, APP and TNF) are directly related to the occurrence of migraine.

Gene enrichment analysis is aimed at functional classification of target genes and determination of the biological significance of phenotypic difference. In biological process enrichment analysis, 265 biological processes were related to the anti-migraine action of ARR, and the top 10 biological processes were considered as the main processes. In order to understand the biological processes associated with the anti- migraine effect of ARR more systematically and comprehensively, KEGG pathway enrichment analysis was carried out. Fifty-two pathways were associated with the anti-migraine potential of ARR, and the top 10 pathways were considered as the main pathways involved in the process. Some studies have reports the relationship between some biological processes or pathways and migraine. With respect to positive regulation of nitric oxide biosynthetic process, ample evidence has indicated that nitric oxide is a causative agent in migraine [20]. A study has revealed that dopamine induces migraine occurrence [21]. In addition, stimulation of dopaminergic neurons has been associated with induction of most symptoms of migraine, with patients showing high sensitivity to dopamine receptor [22]. In the inflammatory mediator regulation of TRP channels pathway, TRPV1 and TRPA1 interfere with the occurrence of migraine by regulating the release of calcitonin 
gene-related peptide $[23,24]$. In the present study, the results obtained from biological process and KEGG pathway enrichment analyses have revealed the molecular mechanisms that underlie the anti-migraine action of ARR. The interactions among biological processes or pathways were obvious, based on the enrichment genes in these biological processes or pathways. Meanwhile, the brain was not the only tissue source of the 71 overlapping genes: some other tissues, such as liver, blood, heart, breast, leukocyte, fibroblast, brain stem, G-protein coupled receptors, and breast cancer, were also involved. In this respect, ARR showed holistic characteristic, which is similar to the characteristics of TCMs used for the treatment of various diseases. This suggests that the molecular mechanism involved in the anti-migraine effect of ARR may not be a single biological process or pathway but an interplay of several pathways/processes.

\section{CONCLUSION}

The molecular mechanism of action involved in the anti-migraine effect of ARR has been systematically and comprehensively investigated using network pharmacology. The results have identified the hub genes, major biological processes, and major pathways involved in the anti-migraine action of ARR. However, the tissue expression of genes related to the process is not limited to the brain. The interactions among biological processes, pathways and multi-tissue expressions of genes show that the action of ARR is holistic, which is consistent with the characteristics of TCMs. These results provide a scientific basis for the clinical application of ARR in migraine therapy.

\section{DECLARATIONS}

\section{Acknowledgement}

This work was financially supported by Fundamental Research Funds for the Central Universities, China (no. SWU118045).

\section{Conflict of interest}

No conflict of interest is associated with this work.

\section{Contribution of authors}

We declare that this work was done by the authors named in this article and all liabilities pertaining to claims relating to the content of this article will be borne by the authors. Yun-Bin
Jiang and Mei Zhong conceived and designed the study. Mei Zhong, Ting Huang and ZhongHua Dai collected the data. Yun-Bin Jiang, XingBao Tao and Rong-Ping Yang analyzed the data, while Yun-Bin Jiang wrote the manuscript. All authors read and approved the manuscript for publication.

\section{Open Access}

This is an Open Access article that uses a funding model which does not charge readers or their institutions for access and distributed under the terms of the Creative Commons Attribution License (http://creativecommons.org/licenses/by/ 4.0) and the Budapest Open Access Initiative (http://www.budapestopenaccessinitiative.org/rea d), which permit unrestricted use, distribution, and reproduction in any medium, provided the original work is properly credited.

\section{REFERENCES}

1. International Headache Society. The international classification of headache disorders (3rd edn). Cephalalgia 2018; 38: 1-211.

2. Dodick DW. Migraine. The Lancet 2018; 391: 1315-1330.

3. Munakata J, Hazard E, Serrano D, Klingman D, Rupnow $M F$, Tierce J, Reed M, Lipton RB. Economic burden of transformed migraine: results from the American Migraine Prevalence and Prevention (AMPP) Study. Headache 2009; 49: 498-508.

4. Sparrow AM, Searles JW. The market for migraine drugs. Nat Rev Drug Discov 2019.

5. Zhou L, Chen $P$, Liu L, Zhang $Y$, Liu $X, W u Y$, Jiang $L$, Cheng $D$, Huang $W$, Pettigrew JC, et al. Systematic review and meta-analysis of traditional Chinese medicine in the treatment of migraines. Am J Chin Med 2013; 41: 1011-1025.

6. Wang $X L$, Jin $L J, X U F X, X u Y P, L i X Y$. Research progress of Chinese herb-Asarum. Asia-Pacific Tradit Med 2013; 9: 68-71.

7. Luo $S$, Wang XD, Kuang $P G$, Jia JP, Yang ZJ, Zhou BY, Yu HF, Chang SY, Ma WY. A clinical study of Yangxueqingnaokeli in the preventive treatment of migraine. Chin J Neurol 2001; 34: 291-294.

8. Ding $X$, Zhao WQ, Cai L. Application research of asarum on modern clinical. Chin J Clin Rational Drug Use 2015; 8: 177-179.

9. Li $S$, Fan TP, Jia W, Lu AP, Zhang WD. Network pharmacology in traditional Chinese medicine. Evid Based Complement Alternat Med 2014; 2014: 138460.

10. Yan E, Song J, Liu C, Hong W. A research on syndrome element differentiation based on phenomenology and mathematical method. Chin Med 2017: 12: 19.

11. Wang S, Tong $Y, N g$ TB, Lao L, Lam JK, Zhang KY, Zhang ZJ, Sze SC. Network pharmacological identification of active compounds and potential actions 
of Erxian decoction in alleviating menopause-related symptoms. Chin Med 2015; 10: 19.

12. da Silva $\mathrm{CH}$, da Silva VB, Resende J, Rodrigues PF, Bononi FC, Benevenuto CG, Taft CA. Computer-aided drug design and $A D M E T$ predictions for identification and evaluation of novel potential farnesyltransferase inhibitors in cancer therapy. J Mol Graph Model 2010; 28: 513-523.

13. Wang L, Tu Z, Sun F. A network-based integrative approach to prioritize reliable hits from multiple genomewide RNAi screens in Drosophila. BMC Genomics 2009; 10: 220.

14. Sutherland HG, Maher BH, Rodriguez-Acevedo AJ, Haupt $L M$, Griffiths $L R$. Investigation of brain-derived neurotrophic factor (BDNF) gene variants in migraine. Headache 2014; 54: 1184-1193.

15. Oliveira AB, Bachi ALL, Ribeiro RT, Mello MT, Tufik S, Peres MFP. Unbalanced plasma TNF- $\alpha$ and IL-12/IL-10 profile in women with migraine is associated with psychological and physiological outcomes. $J$ Neuroimmunol 2017; 313:138-144.

16. Emin Erdal M, Herken $H$, Yilmaz M, Bayazit $Y A$. Significance of the catechol-O-methyltransferase gene polymorphism in migraine. Mol Brain Res 2001; 94: 193 196.

17. Takigawa $H$, Kowa $H$, Nakashima K. No associations between five polymorphisms in COMT gene and migraine. Acta Neurol Scand 2017; 135: 225-230.
18. Chen H, Ji CX, Zhao LL, Kong XJ, Zeng XT. Association between Polymorphisms of DRD2, COMT, DBH, and MAO-A Genes and Migraine Susceptibility: A MetaAnalysis. Medicine 2015; 94: e2012.

19. Huang YL, Wan MY, Liang XS, Liang FR. Effect of acupuncture along affected meridian on the MME gene expression of migraine patients without aura of Ganyang hyperactivity syndrome. Chin J Integr Trad West Med 2015; 35: 294-298.

20. Barbanti P, Egeo G, Aurilia C, Fofi L, Della-Morte $D$. Drugs targeting nitric oxide synthase for migraine treatment. Expert Opin Investig Drugs 2014; 23: 11411148.

21. Sokolov AY, Popova NS, Povarenkov AS, Amelin AV. The role of dopamine in primary headaches. Neurochem J 2018; 12: 324-336.

22. D'Andrea G, D'Amico D, Bussone G, Bolner A, Aguggia $M$, Saracco MG, Galloni E, De Riva V, Colavito D, Leon $A$, et al. The role of tyrosine metabolism in the pathogenesis of chronic migraine. Cephalagia 2013; 33 : 932-937.

23. Kunkler PE, Ballard CJ, Oxford GS, Hurley JH. TRPA1 receptors mediate environmental irritant-induced meningeal vasodilatation. Pain 2011; 152: 38-44.

24. Levy D, Strassman AM, Burstein R. A critical view on the role of migraine triggers in the genesis of migraine pain. Headache 2009; 49: 953-957. 